\section{Cells as probes}

Cell-to-cell interactions are key to many biological processes in normal development as well as in diseases, but getting a handle on them in vivo has been problematic, as most platforms provide static readouts and are unable to follow dynamic processes. Zhao et al. describe an approach to hook up live cells to a fluorescent aptamer probe, allowing such interactions to be followed in real time. The researchers combined fluorescence resonance energy transfer (FRET)-based sensors with intravital microscopy to image mesenchymal stem cells (MSC), which home to areas of inflammation, bone marrow and tumors. Starting with a previously described aptamer sensor for platelet-derived growth factor (PDGF, a chemoattractant of mesenchymal cells), they created a quench FRET sensor (Су3/Cy5) that was attached to biotin, a cell-anchoring moiety. Attachment to cells involved a simple three-step process, depositing 21,000 molecules on the surface of each MSC. After showing that the engineered MSCs sensed PDGF produced by cultured breast cancer cells in vitro, the researchers used confocal and multiphoton imaging to track the migration of the sensors to bone marrow 24 hours after injection into mice. (Nat. Nanotechnol. 6, 524-531, 2011)

\section{Broadly neutralizing anti-influenza mAb}

Studying the structures of antibodies bound to viral proteins provides clues that can direct vaccine design. Now for the first time, Corti et al. report the discovery of a monoclonal antibody $(\mathrm{mAb})$ that neutralizes the two known groups of influenza A viruses and structural features of the mAb's pan-influenza cross-reactivity. To discover the rare cells that produced such a $\mathrm{mAb}$, the authors individually screened 104,000 human plasma cells from eight donors previously found to produce strong antibody responses against a broad range of virus subtypes. This effort yielded a single mAb FI6 that bound all tested recombinant or purified influenza hemagglutinin (HA) proteins. The authors then stained cells that were transfected with recombinant HA genes and neutralized viruses from both influenza groups. FI6 was shown to be effective prophylactically and therapeutically in mice against H1N1 and H3N2 strains of influenza, and in ferrets as a prophylactic against a highly pathogenic $\mathrm{H} 5 \mathrm{~N} 1$ strain. Mutagenesis and structural studies, including co-crystallization of HA proteins from both influenza groups with fragments of an optimized FI6, revealed that the broadly neutralizing activity of the $\mathrm{mAb}$ is related to its ability to bind a particular conserved region. (Science 333, 850-856, 2011)

\section{Isogenic stem cell backgrounds}

Human induced pluripotent stem cells (hiPSCs) derived from patients are increasingly being used to study disease-relevant phenotypes in vitro. Although this approach has created valuable insights into illnesses with rapid and robust manifestations, such as

Written by Laura DeFrancesco, Markus Elsner, Peter Hare \& Craig Mak heart disease or muscular atrophy, diseases with long latency and slow progression are more difficult to study. The subtle in vitro phenotypes of stem-cell models of diseases, such as Parkinson's or Alzheimer's, are easily missed when patient-derived cells are compared with cells from genetically different healthy controls. To overcome this problem, Soldner et al. used pairs of isogenic stem cells that differed only in a specific mutation that confers susceptibility to Parkinson's disease. Using zinc-finger nucleases, the authors engineered such cells by either introducing point mutations in the $\alpha$-synuclein gene of embryonic stem cells derived from healthy individuals or correcting a mutation in hiPSCs from patients with Parkinson's. They showed that differences in gene expression between cells of different genetic backgrounds are larger than those caused by genome editing. (Cell 146, 318-331, 2011)

$M E$

\section{Anti-amyloid immunotherapy for AMD}

Age-related macular degeneration (AMD) is the leading cause of irreversible blindness in the elderly, with no effective therapies for the dry form of the disease. Ding et al. extend studies that have implicated the deposition of amyloid $\beta(A \beta)$ in AMD pathogenesis by demonstrating that systemic administration of a bispecific antibody targeting the two major forms of $A \beta$ protects visual function and preserves the structure of the retinal pigmented epithelium in a mouse model that invokes factors that promote the onset of AMD. These dose-dependent effects were correlated with reductions in the levels of deposits containing $A \beta$ and activated complement components, as well as increases in plasma $A \beta$ concentrations. Immunotherapies targeting $A \beta$ peptides have demonstrated that a reduction in amyloid plaques have improved cognitive function in mouse models of Alzheimer's disease. The identification of $A \beta$ as a viable target for AMD therapy suggests that, regardless of their clinical value for treating Alzheimer's disease, $A \beta$-targeting antibody drugs and vaccines could be repurposed for preventing and/or treating AMD. (Proc. Natl. Acad. Sci. USA 108, E279-E287, 2011)

\section{Spotlight on serine metabolism in cancer}

Two groups have found that the gene encoding the metabolic enzyme phosphoglycerate dehydrogenase (PHDGH), which is involved in serine biosynthesis, is essential in some cancer cells, thereby identifying the serine synthesis pathway as a new potential drug target in cancer. Working with a human breast cancer cell line, Possemato et al. devised an in vivo short hairpin RNA screen to test whether individual candidate metabolic oncogenes are required for tumor formation. From 133 candidates prioritized for screening using bioinformatics, 16 hits were identified. One, $P H G D H$, was studied further because it had been found to be frequently amplified in previous studies of breast cancer and melanoma tissue. Locasale et al. uncovered the importance of serine synthesis and $P H G D H$ using a quantitative metabolic labeling approach. Both groups verified that overexpression of $P H G D H$ is sufficient to drive serine synthesis and required for proliferation of cancer cell lines. Possemato et al. suggest that the oncogenic potential of $P H G D H$ is due in part to its effects on other metabolites in the serine synthesis pathway, such as $\alpha$-ketogluterate, although other mechanisms may be involved because Locasale et al. did not observe changes in $\alpha$-ketogluterate levels. Together these studies reveal how genetic changes to a metabolic gene can promote disease. (Nature 476, 346-350, 2011; Nat. Genet. advance online publication, doi:10.1038/ng.890, 31 July 2011)

CM 